\title{
Assessment of Diverse Quality Metrics for Medical Images Including Mammography
}

\author{
T.Venkat Narayana Rao, \\ Research Scholar, JNTU Kakinada and GNITC, \\ Hyderabad, A.P, India
}

\author{
A. Govardhan, \\ Professor, CSE, JNTUH, \\ Hyderabad, A.P, India
}

\begin{abstract}
This paper presents the comparative analysis of various quality metrics for medical image processing. Measurement of image quality is vital for numerous image-processing applications. Image quality measurement is closely related to image resemblance and assessment in which quality is based on the differences (or similarity) between a degraded image and that of the original image i.e. unmodified image chiefly in mammographic images. We have employed simple verifiable techniques for representing the image quality rather than difficult mathematical procedures, which are costly, time consuming and observer dependent. In this paper, the images have been subjected to various degrees of blur, noise, compression, contrast levels . Bas ed on these factors the quality has been measured in terms of metrics like Mean Squared Error (MSE), Peak Signal-toNoise Ratio (PSNR), Maximum difference (MD) including new metric of image qualities such as Structural Similarity Index Metrics (SSIM)for low cost medical image analy sis.
\end{abstract}

\section{Keywords}

Image quality analysis, Mean Square Error (MSE), Structural Similarity Index Metric(SSIM), Peak Signal to Noise Ratio(PSNR), Mean Absolute Error(MAE)

\section{INTRODUCTION}

Digital images have become very crucial entities in medical analysis and diagnostics for mammography and to investigate scanned image. Digital images are subjected to a broad variety of distortions during acquisition, processing, storage, compression, transmission and reproduction, any of which may effect in a degradation of visual quality [7][1[2]. Identifying the image quality measures that have highest sensitivity to these distortions would help in systematic design of coding, communication and imaging systems to improvise or to optimize the picture quality for a desired quality of service at a minimum cost. For image quality measurement, there are basically two approaches:-

\section{1) Subjective measurements.}

2) Objective measurements.

Subjective measurements are the result of human experts providing their view of the image quality and objective measurements are computed with the mathematical algorithms. In the applications in which images are ultimately to be viewed by human beings is the only exact method of quantifying visual image quality, through subjective evaluation. In reality, subjective evaluation is usually too inconvenient, time-consuming and expensive. The motto of research in objective image quality assessment is to extend quantitative measures that can automatically foresee perceived image quality [2][16].

An objective image quality metric can play a variety of roles in image processing applications. It can be used to dynamically supervise and adjust image quality. This approach could be used to optimize algorithms and parameter settings of image processing systems in a way to assist medical investigation. It can be used to benchmark image processing systems and algorithms [5][8].

Objective image quality metrics can be classified according to the availability of an original (distortion-free) image, with which the distorted image is to be compared. The majority approaches are known as full-reference, this mean that a complete reference image is assumed to be known. In numerous practical applications, the reference image is not accessible, and a no-reference or "blind" quality assessment mechanism is desirable. In a third type of method, the reference image is only partially accessible. It is in the form of a set of extracted features made available as side information to help evaluate the quality of the distorted image. This is denoted as a reduced-reference quality assessment [1][17]. This new method focuses on fullreference image quality assessment.

The simplest and most extensively used full-reference quality metric is the mean squared error (MSE), computed by averaging the squared intensity differences of distorted and reference image pixels. Another popular quantity measure is Peak Signal-to- Noise Ratio (PSNR). These are appealing because they are straightforward to calculate. These measures have clear purpose and meaning and are mathematically suitable in the background of optimization. However, they are not extremely well matched to apparently visual quality. MSE and PSNR lack a critical feature and the ability to assess image similarity across distortion types. In the past three decades, a great deal of attempt one has to put to development of quality assessment methods that take the advantage of known characteristics of the human visual system (HVS)[3][18]. The majority of the proposed perceptual quality assessment models have followed a strategy of modifying the MSE measure so that errors are minimized in agreement with their visibility. Among many of objective measures of picture quality, based on quantifiable distortion measures, the Table I. illustrates the quality metrics available and are frequently used.

\section{POPULAR METHODS TO MEASURE THE QUALITY OF IMAGE}

Where, $\mathrm{x}(i, j)$ represents the original (reference) image and $\mathrm{x}^{\prime}(i, j)$ represents the distorted (modified) image. Two Human visual systems (HVS) based image quality metrics are the universal image quality index $(Q)$ and the Structural Similarity Index (SSIM) [6]. Universal image quality metric: Let $x=\{x i \mid \mathrm{i}=1,2,3, \ldots, \mathrm{N}\}, y=\{y i \mid \mathrm{i}=1,2,3, \ldots, \mathrm{N}\}$ be the original and the test images, respectively. The equation (1) to (8) in this section illustrate the calculation metrics for the Image quality.

$$
Q=\frac{4 \times \sigma_{x y} \quad x \quad \bar{x} \times \quad \bar{y}}{\left(\sigma_{x}^{2}+\sigma_{y}^{2}\right) \times\left((\bar{x})^{2}+(\bar{y})^{2}\right)}
$$


$\bar{x}, \bar{y}, \sigma_{x}^{2}, \sigma_{y}^{2}$, and $\sigma_{x y}$ are given as

$\bar{x}=1 / \mathrm{N} \sum_{i=1}^{N}\left(x_{i}\right)$

$\bar{y}=1 / \mathrm{N} \sum_{i=1}^{N}\left(y_{i}\right)$

$\sigma_{x}^{2}=\frac{1}{\mathrm{~N}-1} \sum_{i=1}^{N}\left(x_{i}-\bar{x}\right)^{2}$

$\sigma_{x y}=\frac{1}{\mathrm{~N}-1} \sum_{i=1}^{N}\left(x_{i}-\bar{x}\right)\left(y_{i}-\bar{y}\right)$

The dynamic range of $\mathrm{Q}$ is $[-1,1]$, with the best value achieved when $y i=x i, i=1,2, \ldots, n$. The index is computed for every window, which leads to a quality map of the image. The total quality index is the average of all the $Q$ values in the quality map

$Q=\frac{1}{M} \sum_{j=1}^{M} Q_{j}$

$M=$ total number of windows.

SSIM (Structural similarity index metric): Q produces unstable results when either $\left((\bar{x})^{2}+(\bar{y})^{2}\right)$ or $\left(\sigma_{x}^{2}+\sigma_{y}^{2}\right)$ is very near to zero. In order to avoid this problem, the measure has been generalized to the Structural Similarity Index (SSIM): [1]

$\sin =\frac{(2 \times \bar{x} \times \bar{y}+C 1)\left(2 \times \sigma_{x y}+C 2\right)}{\left(\sigma_{x}^{2}+\sigma_{y}^{2}+C 2\right) \times\left((\bar{x})^{2}+(\bar{y})^{2}+C 1\right)}$

(7)

$\mathrm{C} 1$ and $\mathrm{C} 2$ are constant. As in the case of $\mathrm{Q}$, the general image quality MSSIM is acquire by computing the average of SSIM values over all windows:

$$
\text { MSSIM }=\frac{1}{M} \sum_{j=1}^{M} \operatorname{sis}_{j}
$$

(8)

$M=$ total number of windows.

\section{THE COMPARATIVE PROPOSED APPROACH}

The evaluation of image quality is the central aim for many image processing systems, such as those for enhancement, compression, transmission and also reproduction. A great deal of effort has gone into designing quality assessment methods that take advantage of known characteristics of the HVS. Natural image signals are highly structured[12]. The most basic principle to image quality assessment is that the HVS is highly adapted to extract structural information from the visual scene, and consequently a measurement of structural similarity (or distortion) provides a good approximation to perceptual image quality. Basing on how structural information and structural distortion are defined, there may be different means to develop image quality assessment algorithms [6][19]. Following steps are taken in proposed approach: 1. Study the existing metrics. 2. Simulate the various quality metrics. 3. Execute the various quality metrics by augmenting noise, blur, compression, and contrast applications with medical images such as mammographic and MRI etc.

\section{RESULTS AND FINDINGS}

The functions illustrated in table I are being evaluated with diverse inputs as shown. Based on this a comparative evaluation of various quality metrics has been done with three different kinds of images, which are commonly used in areas of medical image processing:

A. MRI image (noise removal and with compression)

B. X-Ray image (for blur image applications)

C. Ultra sound image (with contrast application)

MRI image has been chosen for application of noise[3][9]. These types of images have small size particulars, which are vulnerable to random noise[12][14]. Gaussian noise has been selected for adding noise to the image. In Gaussian Noise, power is directly proportional to the variance. Therefore, different values of noise variances are taken for adding different level of noise powers to the image. The images corresponding to diverse noise powers are shown in Figure 1 and values of various quality metrics is shown in Table 2 .

As can be seen from Table II, all quality metrics acknowled ge the changes to the noise powers variation. MD appears to be the mostly sensitive metric whereas SC \& PMSE being the slightest sensitive to the variation in noise power. From the Tables it is obvious that SSIM give results with greater accuracy.
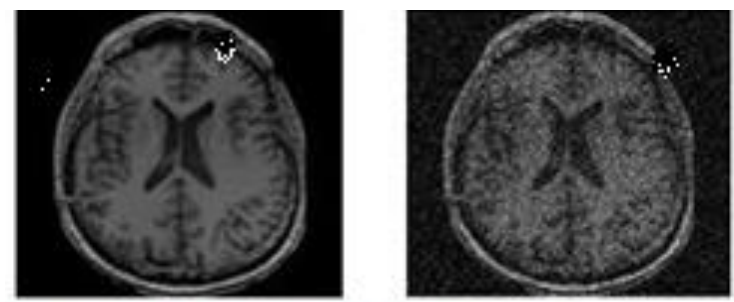

a) Original MRI image b) Noisy (Varianca=02)
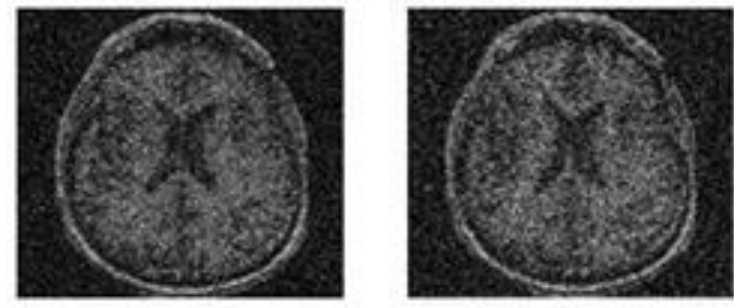

c) Noisy (Varianou=04) d) Noisy (Varianch=,05)
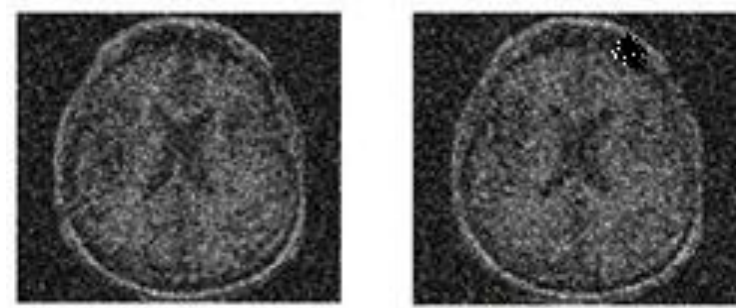

4) Noisy (VariancF=, 0S?

6) Noisy (Variase, 1)।

Figure 1. Different MRi Images with variance

Table 3 and Figure 1 show different values of quality metrics for MRI compression image. The compression depend upon the block processing, means image is bigger we can use smaller block of image at a time. To compress the image, the first step is to subdivide the input image into non-overlapping pixel blocks of size $8 \times 8$ [10][13]. They are subsequently processed left to right, top to bottom. The two-dimensional DCT is calculated for every block. The DCT coefficients are then quantized, coded and transmitted. By applying block, processing image signal firstly converted from space domain 
to frequency domain, then mask as a frequency domain filter. By multiplying frequency domain filter to frequency, domain signal and we get compression signal, which will depend upon the number of elements in the filter. Table III depicts that by decreasing no. of elements are less in filter percentage of compression is changes [4].

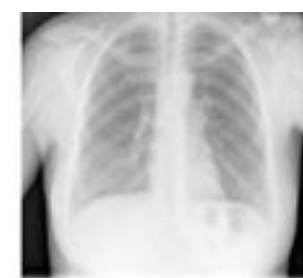

a) Original $x$-ray image

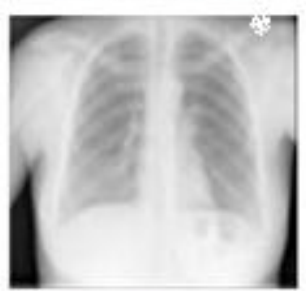

c) Blur (Variance=4)

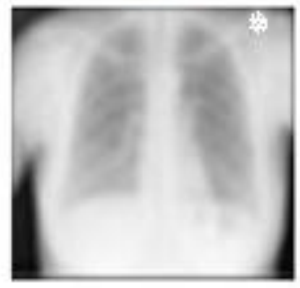

e) Blur (Variance $=10$ )
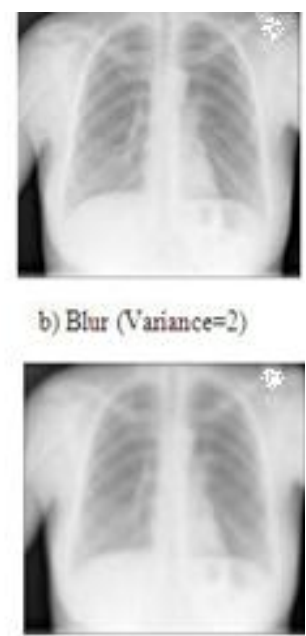

d) Blur (Variance $=\$$ )

f) Blur (Variance $=12)$ b) Blur (Variance=2)

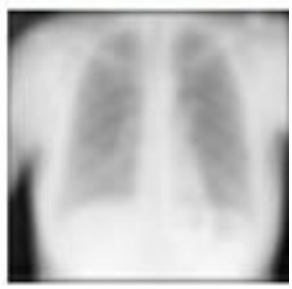

Figure 2. Different x-ray blurred variance images

The images corresponding to different degrees of blur have been shown in Figure 2 and values of various quality metrics is shown in Table IV. From the table it is understandable that most of the quality metrics admit the change in degree of blur except NK, SC , MD, and PMSE. So MSE, AD, M AE, PSNR, IF and SSIM are additional significant whereas MD, NK, SC and PMSE have little significance in image blur applications. As depicted in Table IV it is apparent that SSIM give results with greater consistency [5] [8][11]. The Contrast of an image shows the distribution of pixel values of an image from minimum to maximum values. A low contrast image has this distribution confined in a small range whereas a high contrast image has pixel values distribution over the full range from minimum to maximum probable pixel value. We have chosen an ultrasound image for variation in its contrast. Contrast of the image has been varied with the help of function, which is inherent in MATLAB. It changes the limiting range of pixel of output image as per specification in its arguments as shown in figure 3 .
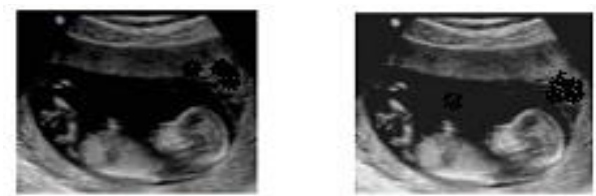

a) Otiginal ultrasound image

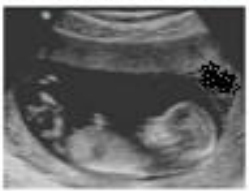

b) Contrast (Stretching limits $-0.1-0.9$ )

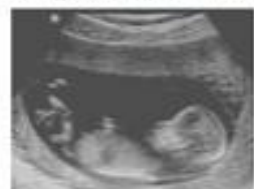

c) Contrast (Stretching limits $=0.2 .0 .5$ ) d) Contrast (Stretching limits $=0.3-0.7$ )

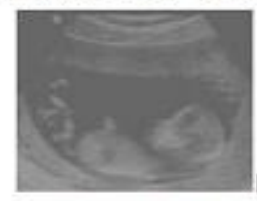

e) Coetrast (Stretching limits $=0.4-0.6$ )

Figure 3. Ultrasound Images with limits.

From the Table 4 it is clear that most of the quality metrics recognize the change in degree of contrast except NK and IF. So MSE, PSNR, AD, MD, MAE, PMSE and SSIM are more significant whereas, NK, IF and SC have little significance in ultrasound image contrast applications. As can be seen from the table SSIM has elevated accuracy of results and grater values of SSIM indicate greater image similarity 1[5][17].

\section{CONCLUSION}

In this paper, comparative analysis of various quality metrics has been explored. Three different kinds of medical application images have been processed for different levels of compression, noise powers, blur and contrast. All image quality metrics described in this paper have been calculated for all four types of image processing operations. Output results have been evaluated for comparison of various quality metrics. These results show that only a section i.e. a subset of quality metrics is suited for a particular type of image processing operation except the SSIM metric which is capable of expressing image quality irrespective of the kind of operation. Other quality metrics are easy but not accurate for all kinds of image processing operations. SSIM has high performance, works precisely and provides a high-quality approximation of quality measurement but with a moderately bigger computational time. The above work can be applied to meticulously to analyze much impending breast cancer through mammographic diagnosis and detection cancer of type I and II with a lower cost for early detection.

\section{REFERENCES}

[1] Z.Wang, A.C Bovik, H.R Sheikh, and E.P Simoncelli, 2004. Image Quality Assessment: From Error Visibility to Structural Similarity. IEEE Transactions of Image Processing, (April 2004), vol. 13 1-12.

[2] Zhou Wang and Alan C. Bovik, 2002. A Universal Image Quality Index. IEEE Signal Processing Letters, vol. 9, No. 3, 81-84.

[3] Z. Wang, A. C. Bovik, and L. Lu, 2002. Why is image quality assessment so difficult. In Proceedings of IEEE Int.Conf. Acoustics, Speech, and Signal Processing, (May 2002), vol. 4. 3313-3316. 
[4] Eric Silva, Karen A. Panetta, Sos S. Agaian, 2007. Quantify similarity with measurement of enhancement by entropy, Proceedings: Mobile Multimedia/Image Processing for Security Applications, SPIE Security Symposium (April 2007), Vol. 6579, 1.

[5] Z. Wang, H. R. Sheikh, and A. C. Bovik, 2003. Objective video quality assessment. The Handbook of Video Databases: Design and Applications, B. Furht and O. Marques, Eds. Boca Raton, FL: CRC Press,.

[6] Alan C. Brooks, Xiaonan Zhao, and Thrasyvoulos N. Pappas, 2008 .Structural Similarity Quality Metric in a Coding Context: Exploring the Space of Realistic Distortions, IEEE Transactions on image processing, (August 2008)Vol. 17, No. 8.

[7] A. M. Eskicioglu and P. S. Fisher, 1995 . Image quality measures and their performance, IEEE Trans. Commun.,( Dec 1995), vol. 43, 2959-2965.

[8] Sonja Grgic , Mislav Grgic,and Marta Mrak, 2004. Reliability Of Objective Picture Quality Measures, Journal of Electrical Engineering, (October 2004), Vol. 55, No. 1-2.

[9] A. M. Eskicioglu and P. S. Fisher, 1995 . Image quality measures and their performance, IEEE Trans. Commun.,( Dec 1995), vol. 43, 2959-2965.

[10] Sonja Grgic , Mislav Grgic,and Marta Mrak, 2004. Reliability Of Objective Picture Quality Measures, Journal of Electrical Engineering, (October 2004), Vol. 55 , No. $1-2$.

[11] Oliver, A., Lladó, X., Pérez, E., J., P., Denton, E., Freixenet, J., et al. (2010). A Statistical Approach for Breast Density Segmentation. J Digit Imaging, 23, No.5.
International Journal of Computer Applications (0975 - 8887) Volume 83 - No4, December 2013

[12] Sankar, D., \& Thomas, T. (2010, Oct). A New Fast Fractal Modeling Approach for the Detection of Microcalcifications in Mammograms. J Digit Imaging. 2010 Oct;23(5):538-46. Epub 2009 Jul 18. , 23, N.5, pp. 538-546.

[13] Wang, X., Lederman, D., Tan, J., \& Zheng, B. (2010). Computer-aided Detection: The Impact of Machine Learning Classifier and Image Feature Selection on Scheme Performance. IJIIP: International Journal of Intelligent Information Processing, 1, No 1.

[14] Schulz-Wendtland, R., Fuchsjäger, M., Wackerc, T., \& Hermannd, K. (2009). Digital mammography: An update. European Journal of Radiology , 72, pp. 258-265.

[15] Sampat, M., Markey, M., \& Bovik, A. (2005). ComputerAided Detection and Diagnosis in Mammography. In A. Bovik, Handbook of Image and Video Processing (pp. 1195-1217). Elsevier.

[16] Masala, G. (2006). Computer Aided Detection on Mammography. World Academy of Science, Engineering and Technology , 15.

[17] Khoo, L., Taylor, P., \& Given-Wilson, R. (2005). Computer-aided Detection in the United Kingdom National Breast Screening Programme: Prospective Study. Radiology , 237, pp. 444-449.

[18] American Cancer Society; "Breast Cancer Facts \& Figures”, Inc. 2009-2010.

[19] H.B.Kekre, T. K. Sarode, S. M. Gharge; "Tumor Detection in Mammography Images using Vector Quantization Technique", International Journal of Intelligent Information Technology Application, 332(5):237-242. 2009.

Table I. Conventional and new Metrics of Quality

\begin{tabular}{|l|l|l|}
\hline & TYPE & DESCRIPTION \\
\hline 1. & MSE(Mean Square Error) & $\frac{1}{M N} \sum_{i=1}^{M} \sum_{j=1}^{N}(x(i, j)-y(i, \mathrm{j}))^{2}$ \\
\hline 2. & $\begin{array}{l}\text { PSNR(Peak Signal to Noise } \\
\text { Ratio) }\end{array}$ & $\boldsymbol{P S N R}=\mathbf{1 0} \log _{10} \frac{\left(2^{n}-\mathbf{1}\right)^{2}}{\sqrt{M S E}}$ \\
\hline 3. & AD(Average Deviation) & $A D=\frac{1}{M N} \sum_{i=1}^{M} \sum_{j=1}^{N}(x(i, j)-y(i, j))$ \\
\hline 4. & MD(Maximum Difference) & $\mathrm{MD}=\operatorname{MAX} \mid(x(i, j)-y(i, j) \mid$ \\
\hline 5. & MAE(Mean Absolute Error) & MAE $=\frac{1}{M N} \sum_{i=1}^{M} \sum_{j=1}^{N} \mid(x(i, j)-y(i, j) \mid$ \\
\hline
\end{tabular}




\begin{tabular}{|c|c|c|}
\hline & TYPE & $\overline{\text { DESCRIPTION }}$ \\
\hline 6. & NK(Normal Cross-Correlation) & $N K=\frac{\sum_{i=1}^{M} \sum_{j=1}^{N}((x(i, j)-y(i, j))}{\sum_{i=1}^{M} \sum_{j=1}^{N}(x(i, j))^{2}}$ \\
\hline 7. & SC(Structural Content) & $S C=\frac{\sum_{i=1}^{M} \sum_{j=1}^{N}(y(i, j))^{2}}{\sum_{i=1}^{M} \sum_{j=1}^{N}(x(i, j))^{2}}$ \\
\hline 8. & IF(Image Fiedelity) & $I F=1-\frac{\sum_{i=1}^{M} \sum_{j=1}^{N}(x(i, j)-y(i, j))^{2}}{\sum_{i=1}^{M} \sum_{j=1}^{N}(x(i, j))}$ \\
\hline 9. & $\begin{array}{lll}\text { PMSE(peak } & \text { Mean } & \text { Square } \\
\text { Error) } & & \\
\end{array}$ & $P M S E=\frac{1}{M N} \times \frac{\sum_{i=1}^{M} \sum_{j=1}^{N}(x(i, j)-y(i, j))^{2}}{\left(\operatorname{MAX}(x(i, j))^{2}\right.}$ \\
\hline 10. & $\begin{array}{ll}\text { SSIM(Structural } & \text { Similarity } \\
\text { Index Metrics) } & \end{array}$ & $\operatorname{SSIM}=\frac{(2 \times \bar{x} \times \bar{y}+C 1)\left(2 \times \sigma_{x y}+C 2\right)}{\left(\sigma_{x}^{2}+\sigma_{y}^{2}+C 2\right) \times\left((\bar{x})^{2}+(\bar{y})^{2}+C 1\right)}$ \\
\hline
\end{tabular}

Table 2. Comparison of Quality Metrics for Noisy MRI Image

\begin{tabular}{|l|l|l|l|l|l|l|l|l|l|l|}
\hline Variance & MSE & PSNR & AD & MD & MAE & NK & SC & IF & PMSE & SS IM \\
\hline 0.02 & 58.4048 & 39.2986 & -6.6495 & 153 & 22.4545 & 1.0146 & 1.3525 & .7066 & .0012 & .2036 \\
\hline 0.04 & 63.9898 & 39.1042 & -10.0501 & 190 & 30.5875 & 1.0263 & 1.5972 & .4554 & .0012 & 0.1327 \\
\hline 0.06 & 65.2490 & 39.0579 & -13.766 & 239 & 36.6935 & 1.0598 & 1.9323 & .2172 & .0013 & .1072 \\
\hline 0.08 & 66.5353 & 39.0155 & -16.6531 & 255 & 41.4679 & 1.0829 & 2.1662 & 0.0084 & .0014 & .0882 \\
\hline 0.1 & 68.3621 & 38.9567 & -18.5784 & 255 & 45.1500 & 1.0900 & 2.3725 & -0.1925 & .0013 & .0741 \\
\hline
\end{tabular}

Table 3. Comparison of Various Quality Metrics for Compressed MRI Image

\begin{tabular}{|c|c|c|c|c|c|c|c|c|c|c|}
\hline Compression \% & MSE & PS NR & AD & MD & MAE & NK & SC & IF & PMSE & SS IM \\
\hline 15 & 42.1701 & 40.0568 & .1255 & 162 & 10.7485 & .8843 & .8845 & .8852 & $7.9233 \mathrm{E}-04$ & .5804 \\
\hline 13 & 29.1606 & 40.8143 & -.1179 & 152 & 7.1392 & .9443 & .9416 & .9459 & $5.5913 \mathrm{E}-04$ & .7564 \\
\hline 11 & 20.7699 & 41.5415 & -.0837 & 137 & 5.0232 & .9681 & .9617 & .9694 & $3.9982 \mathrm{E}-04$ & .8579 \\
\hline 9 & 14.5008 & 42.1790 & -.0326 & 114 & 3.7552 & .9806 & .9776 & .9818 & $2.9813 \mathrm{E}-04$ & .9184 \\
\hline 7 & $\mathbf{1 2 . 0 4 2 6}$ & $\mathbf{4 2 . 9 1 5 4}$ & $\mathbf{- . 0 1 4 8}$ & $\mathbf{9 2}$ & $\mathbf{2 . 7 9 1 5}$ & $\mathbf{. 9 8 8 5}$ & $\mathbf{. 9 8 6 5}$ & $\mathbf{. 9 8 8 5}$ & $\mathbf{2 . 1 2 4 1 E - 0 4}$ & $\mathbf{. 9 5 1 8}$ \\
\hline
\end{tabular}


Table 4. Comparison of Various Quality Metrics For dissimilar Contrasts of Ultrasound Image

\begin{tabular}{|c|c|c|c|c|c|c|c|c|c|c|}
\hline $\begin{array}{l}\text { Stretch } \\
\text { limits }\end{array}$ & MSE & PS NR & AD & MD & MAE & NK & $\begin{array}{l}\text { S } \\
\text { C }\end{array}$ & IF & PMSE & SS IM \\
\hline $0.1-0.9$ & .00289 & 60.8371 & -31.3843 & 45 & 31.3858 & 1.3491 & 1.8728 & .8241 & $5.1434 \mathrm{E}-08$ & .6849 \\
\hline $0.2-0.8$ & .2238 & 51.3915 & -41.5258 & 51 & 41.5541 & 1.3248 & 1.9363 & 1.0040 & $3.7094 \mathrm{E}-06$ & .6130 \\
\hline $0.3-0.7$ & 1.9789 & 46.6198 & -52.1702 & 79 & 52.5986 & 1.2892 & 2.1567 & .4233 & $3.5211 \mathrm{E}-05$ & .5215 \\
\hline $0.4-0.6$ & 10.607 & 43.1039 & -62.4971 & 101 & 64.9350 & 1.2503 & 2.4578 & 1.0030 & $1.7661 \mathrm{E}-04$ & .3726 \\
\hline
\end{tabular}

